\title{
Phases Transitions in a Cross-Cavity Quantum Rabi Model Possessing PT Symmetric Structure
}

\author{
Ning Wang ${ }^{1}$, Zhi-Rui Gong ${ }^{2}$, Jing Lu ${ }^{1 *}$ and Lan Zhou ${ }^{1}$ \\ ${ }^{1}$ Key Laboratory of Low-Dimensional Quantum Structures and Quantum Control of Ministry of Education, Department of \\ Physics and Synergetic Innovation Center of Quantum Effects and Applications, Hunan Normal University, Changsha, China, \\ ${ }^{2}$ College of Physics and Optoelectronic Engineering, Shenzhen University, Shenzhen, China
}

We investigated the quantum phase transitions in a cross-cavity quantum Rabi model which possesses the parity-time symmetric structure. Both the dissipative quantum Rabi phase transition and the spontaneous parity-time symmetry breaking exist simultaneously in such system. The competition between these two phase transitions produces the plentiful phase diagram. We also propose the detection of the dissipative cross-cavity quantum Rabi phase transition through the measurement of the parity-time symmetric or asymmetric states, which shed light on the precise detection of the few-body phase transition.

OPEN ACCESS

Edited by:

Yanpeng Zhang,

Xi'an Jiaotong University, China

Reviewed by:

Qing Ai,

Beiiing Normal University, China

Youbin Yu,

Zhejiang Sci-Tech University, China

*Correspondence:

Jing Lu

lujing@hunnu.edu.cn

Specialty section:

This article was submitted to

Optics and Photonics,

a section of the journal

Frontiers in Physics

Received: 04 July 2019

Accepted: 22 August 2019

Published: 10 September 2019

Citation:

Wang N, Gong Z-R, Lu J and Zhou L

(2019) Phases Transitions in a

Cross-Cavity Quantum Rabi Model

Possessing PT Symmetric Structure.

Front. Phys. 7:127.

doi: 10.3389/fphy.2019.00127
Keywords: phases transitions, cross-cavity, quantum Rabi model, PT symmetric, PT symmetry breaking PACS numbers: 42.50.-p, 03.67.Lx

\section{INTRODUCTION}

Quantum Rabi model (QRM) describing the coupling between one two-level atom and one quantized cavity's electromagnetic mode provides the simplest paradigm of the quantum lightmatter interaction $[1,2]$, which plays crucial role in many areas of the modern physics such as quantum optics [3-5], quantum information processing [6-9], and condensed matter science [1014]. Under the rotating wave approximation to neglect the counter-rotating terms [15], quantum Rabi model (a.k.a. Jaynes-Cummings model) can be readily solved for the near resonance or the weak coupling parameter regions in many experiments. Thanks to the development of the experimental techniques, the ultra-strong and deep-strong coupling regime can be accessed in recent experiments [16-23]. In these regimes, the counter-rotating terms can not be neglected any more which results in several intriguing phenomenon. One of them is the finite-component system phase transition, where the ground state experiences a non-analytic change in the ultrastrong atomcavity-mode coupling together with large detuning [24-31]. The quantum phase transition in an dissipative quantum Rabi model has also been studied for a realistic purpose, where the cavity emits photons at a certain decay rate. The quantum Rabi model just provides a promising platform to investigate the physics of the phase transition in quantum optical systems, which has already attracted a lot of interest. If another kind of the phase transition is introduced in such system, the competition between different phase transition mechanisms is supposed to lead to plentiful phase diagram.

On the other hand, parity-time (PT) symmetric structure is also an intriguing topic in quantum optical system [32-34]. A system with PT symmetry can undergo a phase transition at the exceptional point, where the eigenvalues of the non-Hermitian Hamiltonian varies from real values to complex values when crossing the exceptional point [35-37]. The PT symmetry breaking actually results in various interesting phenomenon studied both theoretically and experimentally 
such as non-reciprocal photonic transmission [38], enhancement of nonlinear interaction [39, 40], loss-induced or gaininduced transparency [41, 42], efficient photon or phonon lasing [43-47], ultralow-threshold optical chaos [48, 49], quantum metrology [50], and so on. If PT symmetric structure can be introduced in the QRM, two phase transition mechanisms actually compete with each other and provide plentiful phase diagram. Such system can be experimentally implemented by the whispering-gallery modes in the ring cavities or the superconducting transmission lines coupling to one Cooper pair box.

In this paper, we investigate the plentiful phase transitions and phase diagram of the cross-cavity quantum Rabi model together with PT symmetric structure. Since the PT symmetric structure requires a balanced loss and gain on different cavities, the two cavities coupling to the same atom as the simplest model has been taken into consideration. We find that the competition between the quantum Rabi phase transition and the PT symmetry breaking results in plentiful phase transitions and phase diagram. The normal phase and superradiant phase emerging in the previous dissipative quantum Rabi model still exist in our system. The effective coupling is inversely proportional to $g^{2}$ in the superradiant phase instead of being proportional to $g^{2}$ in the normal phase. In contrast, the PT asymmetric phase and symmetric phase can also affect the dissipative quantum Rabi model. For the PT asymmetric phase, the total system has steady state when the time tends to infinite because the energy will eventually dissipate into the surrounding environment. When the PT asymmetric phase changes to a symmetric one, the gain and loss of the system can be rebalanced and the original dissipative quantum Rabi model behaves like a decay free Rabi model. It means that except the normal phase and superradiant phase existing in the previous dissipative quantum Rabi model, an oscillating phase emerges in the current system, which exactly is attributed from the PT symmetric structure. It also changes the PT symmetry breaking from a second-order phase transition to a first order one.

The paper is organized as follows. In section The Dissipative QRM Together With PT Symmetric Structure, we introduce the cross-cavity QRM together with PT symmetric structure. We perform a semiclassical analysis of the model to show the steady state and the oscillating state. In section Dissipative Phase Transition, we present a full quantum mechanical solution for the PT symmetry breaking. The competition between the two phase transition mechanisms results in a plentiful phase diagram, which is shown and discussed in section Dissipative Phase Transition. We also propose a self-consistent equation. Finally, we conclude our paper in section Conclusion.

\section{THE DISSIPATIVE QRM TOGETHER WITH PT SYMMETRIC STRUCTURE}

In this article, we consider the dissipative cross-cavity quantum Rabi model (QRM) together with parity-time (PT) symmetric structure (shown in Figure 1), which is described by a master equation as

$$
\dot{\rho}=-i\left[H_{0}, \rho\right]+\mathcal{L}[\rho]
$$

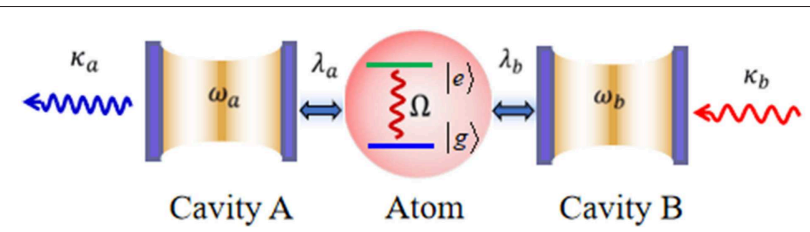

FIGURE 1 | The schematics of the dissipative QRM together with PT symmetric structure when $\omega_{a}=\omega_{b}=\omega_{0}, \lambda_{a}=\lambda_{b}=\lambda$, and $\kappa_{a}=\kappa_{b}=\kappa$.

where Hamiltonian $(\hbar=1)$

$H_{0}=\omega_{a} a^{\dagger} a+\omega_{b} b^{\dagger} b+\frac{\Omega}{2} \sigma_{z}-\lambda_{a}\left(a+a^{\dagger}\right) \sigma_{x}-\lambda_{b}\left(b+b^{\dagger}\right) \sigma_{x}$,

governs the coherent dynamics of the cross-cavity QRM with the annihilation (creation) operator of the two cavity modes $a\left(a^{\dagger}\right)$ and $b\left(b^{\dagger}\right), \sigma_{x}$ and $\sigma_{z}$ are Pauli matrices for a two-level system. Here, $\Omega$ is the qubit transition frequency and $\lambda_{a(b)}$ is the coupling strength between the first (second) cavity and the atom. The dissipative nature of the cross-cavity including the loss of the first cavity and the gain of the second cavity is described by Lindblad operators as $\mathcal{L}[\rho]=\mathcal{L}_{a}[\rho]+$ $\mathcal{L}_{b}[\rho]$ with $\mathcal{L}_{a}[\rho]=\kappa_{a}\left(2 a \rho a^{\dagger}-\rho a a^{\dagger}-a a^{\dagger} \rho\right)$ and $\mathcal{L}_{b}[\rho]=$ $\kappa_{b}\left(2 b^{\dagger} \rho b-\rho b^{\dagger} b-b^{\dagger} b \rho\right)$. The oscillator frequencies of the cavity modes and the coupling strengths are tuned to the same one as $\omega_{a}=\omega_{b}=\omega_{0}$ and $\lambda_{a}=\lambda_{b}=\lambda$, and the loss of the first cavity and the gain of the second cavity are symmetric as $\kappa_{a}=\kappa_{b}=\kappa$ in order to introduce the PT symmetry into the dissipative cross-cavity QRM. Although the PT symmetric and asymmetric phases can also exist when the frequencies, the coupling strengths and the loss (gain) of cavities are not identical, in the sake of the simplicity we consider the identical parameters of the cavities in the following discussion.

By applying the same semi-classical analysis in Hwang et al. [30], in the limit of extremely large detuning $\Omega / \omega_{0} \gg 1$ and ultra-strong coupling $\lambda / \omega_{0} \gg 1$, the quantum fluctuations are neglected and the mean-field properties are captured. From the standard Heisenberg-Langevin equation of motion acquired from the master equation in Equation (1) where the quantum fluctuations and the correlations between the operators are neglected, a set of semiclassical equation of motion of the dissipative cross-cavity QRM together with the PT symmetry as

$$
\begin{aligned}
\langle\dot{a}\rangle= & -i\left(\omega_{0}-i \kappa\right)\langle a\rangle+i \lambda\left(\left\langle\sigma_{+}\right\rangle+\left\langle\sigma_{-}\right\rangle\right), \\
\langle\dot{b}\rangle= & -i\left(\omega_{0}+i \kappa\right)\langle b\rangle+i \lambda\left(\left\langle\sigma_{+}\right\rangle+\left\langle\sigma_{-}\right\rangle\right), \\
\left\langle\dot{\sigma_{+}}\right\rangle= & i \Omega\left\langle\sigma_{+}\right\rangle+i \lambda(\langle a\rangle \\
& \left.+\langle a\rangle^{*}+\langle b\rangle+\langle b\rangle^{*}\right)\left\langle\sigma_{z}\right\rangle, \\
\left\langle\dot{\left.\sigma_{z}\right\rangle}=\right. & i 2 \lambda\left(\langle a\rangle+\langle a\rangle^{*}\right. \\
& \left.+\langle b\rangle+\langle b\rangle^{*}\right)\left(\left\langle\sigma_{+}\right\rangle-\left\langle\sigma_{-}\right\rangle\right) .
\end{aligned}
$$

If there is only pure dissipation in the system, the solution of the above semiclassical equation eventually tends to a steady 
one when the evolution time is much larger than the dissipative time $\kappa^{-1}$. However, this is not always true in a system with PT symmetric structure because both loss and gain simultaneously exist. It depends on whether the spontaneous symmetry breaking of the PT symmetry occurs or not. If the system stays at the PT asymmetric state, the total energy still dissipate into the surrounding environment of the first cavity even the second cavity gains energy from its surrounding environment and the above semiclassical equation has steady solution. While at the PT symmetric state, the energy loss in the first cavity and the energy gain in the second cavity achieve delicate balance and an oscillating solution possibly exist in our cross-cavity QRM. In this sense, the phase transitions could be more plentiful in our model.

Firstly, the steady-state solution should satisfy

$$
\begin{aligned}
& 0=\left(1-i \frac{\kappa}{\omega_{0}}\right) \alpha-\frac{g}{2}\left(S_{+}+S_{+}^{*}\right), \\
& 0=\left(1+i \frac{\kappa}{\omega_{0}}\right) \beta-\frac{g}{2}\left(S_{+}+S_{+}^{*}\right), \\
& 0=S_{+}+\frac{g}{2}\left(\alpha+\alpha^{*}+\beta+\beta^{*}\right) S_{z}, \\
& 0=g\left(\alpha+\alpha^{*}+\beta+\beta^{*}\right)\left(S_{+}-S_{+}^{*}\right) .
\end{aligned}
$$

where $\alpha \equiv\langle a\rangle_{s} / \sqrt{\eta}$ and $\beta \equiv\langle b\rangle_{s} / \sqrt{\eta}$ are renormalized steady-state mean amplitude of the cavity modes, and $S_{+} \equiv$ $\left\langle\sigma_{+}\right\rangle_{s}$ and $S_{z} \equiv\left\langle\sigma_{z}\right\rangle_{s}$ are the steady-state qubit expectation values. Here, we introduce a dimensionless coupling constant $g=2 \lambda / \sqrt{\omega_{0} \Omega}$ and a frequency ratio $\eta \equiv \Omega / \omega_{0}$ for computational convenience.

The following constrain $4\left|S_{+}\right|^{2}+S_{z}^{2}=1$ is introduced due to the conservation of the classical pseudo-spin. Interestingly, the steady solution of the dissipative cross-cavity QRM still exhibits a bifurcation at $g=g_{c}$ with

$$
g_{c}=\sqrt{\frac{1}{2}\left(1+\frac{\kappa^{2}}{\omega_{0}^{2}}\right)}
$$

Below the critical point $\left(g<g_{c}\right)$, we get the trivial solution

$$
\alpha=0, \beta=0, S_{ \pm}=0, S_{z}=-1,
$$

as the normal phase solution with zero mean field amplitude of cavity modes and the ground state of the pseudo-spin. Above the critical point $\left(g>g_{c}\right)$, we get the superradiant phase solution as

$$
S_{z}=-\frac{g_{c}^{2}}{g^{2}}, S_{ \pm}= \pm \frac{1}{2} \sqrt{1-\left(\frac{g_{c}}{g}\right)^{4}}, \alpha=\beta^{*}=\frac{g}{1-i \frac{k}{\omega_{0}}} S_{+},
$$

where cavity modes have nonzero mean field amplitude and the pseudo-spin stays at an excited state as well. Such phase transition of the dissipative cross-cavity QRM exactly inherit from the dissipative QRM. Additionally, $\alpha=\beta^{*}$ still implies the PT symmetric structure here.
Now the oscillating solution is taken into consideration. Where the cavity modes oscillate at frequency $\omega$, the $x$ and $y$ component of the qubit should eventually oscillate at the same frequency $\omega$ along the $z$-axis because the coupling in Equation (2) effectively provide an oscillating driven magnetic field in the $x$-direction. Therefore the oscillating solution should satisfy $\langle\dot{a}\rangle=i \omega\langle a\rangle,\langle\dot{b}\rangle=i \omega\langle b\rangle,\left\langle\sigma_{ \pm}\right\rangle=\mp i \omega\left\langle\sigma_{ \pm}\right\rangle$. For a conserved classical pseudo-spin rotating along $z$-axis, the $z$-component of the pseudo-spin can be steady as $\left\langle\dot{\sigma}_{z}\right\rangle=0$. However, the oscillating frequency can not be simply determined. The oscillating solution exists due to the unbroken PT symmetry, while the PT symmetry breaking is not clear under the current semi-classical treatment. In the next section, we will try to find a self-consistent equation to obtain the oscillating frequency. The Heisenberg-Langevin equation can be rewritten as

$$
\begin{aligned}
i \omega\langle a\rangle= & -i\left(\omega_{0}-i \kappa\right)\langle a\rangle+i \lambda\left(\left\langle\sigma_{+}\right\rangle+\left\langle\sigma_{-}\right\rangle\right), \\
i \omega\langle b\rangle= & -i\left(\omega_{0}+i \kappa\right)\langle b\rangle+i \lambda\left(\left\langle\sigma_{+}\right\rangle+\left\langle\sigma_{-}\right\rangle\right), \\
\mp i \omega\left\langle\sigma_{ \pm}\right\rangle= & i \Omega\left\langle\sigma_{+}\right\rangle+i \lambda(\langle a\rangle \\
& \left.+\langle a\rangle^{*}+\langle b\rangle+\langle b\rangle^{*}\right)\left\langle\sigma_{z}\right\rangle, \\
0= & i 2 \lambda\left(\langle a\rangle+\langle a\rangle^{*}\right. \\
& \left.+\langle b\rangle+\langle b\rangle^{*}\right)\left(\left\langle\sigma_{+}\right\rangle-\left\langle\sigma_{-}\right\rangle\right) .
\end{aligned}
$$

If the oscillator frequencies of the cavity modes and the qubit transition frequency are shifted by $\omega$ as $\omega_{0}^{\prime}=\omega_{0}+\omega$ and $\Omega^{\prime}=\Omega+\omega$, the above set of equations have the same form of the Equations (3a-3d). It means even for the oscillating solution there exhibits a bifurcation at $g^{\prime}=g_{c}^{\prime}$ with

$$
g^{\prime}=\frac{2 \lambda}{\sqrt{\left(\omega_{0}+\omega\right)(\Omega+\omega)}}, g_{c}^{\prime}=\sqrt{\frac{1}{2}\left(1+\frac{\kappa^{2}}{\left(\omega_{0}+\omega\right)^{2}}\right)} .
$$

Below the new critical point $\left(g^{\prime}<g_{c}^{\prime}\right)$, the oscillating solution will degenerate to a normal phase as

$$
\langle a\rangle=0,\langle b\rangle=0,\left\langle\sigma_{+}\right\rangle=0,\left\langle\sigma_{-}\right\rangle=-1
$$

Above the new critical point $\left(g^{\prime}>g_{c}^{\prime}\right)$, the oscillating solution also bifurcates into two stable solutions as

$$
\begin{aligned}
& \left\langle\sigma_{z}\right\rangle=-\left(\frac{g_{c}^{\prime}}{g^{\prime}}\right)^{2},\left\langle\sigma_{+}\right\rangle= \pm \frac{1}{2} \sqrt{1-\left(\frac{g_{c}^{\prime}}{g^{\prime}}\right)^{4}}, \\
& \langle a\rangle=\left\langle b^{\dagger}\right\rangle=\frac{g^{\prime}}{1-i \frac{\kappa}{\omega_{0}+\omega}}\left\langle\sigma_{+}\right\rangle,
\end{aligned}
$$

which actually corresponds to a new phase where both the cavity modes and the pseudo-spin eventually oscillates all the time when the energy loss and the gain are delicate balanced. We will call it oscillating phase and determine the oscillating frequency in the next section.

\section{DISSIPATIVE PHASE TRANSITION}

In this section, we derive an effective master equation for the cross-cavity modes when tracing out the qubit degree of freedom 
in the limit of extremely large detuning $\Omega / \omega_{0} \gg 1$. Based on the Heisenberger-Langevin approach, the quantum dynamics of the system can be investigated in details together with the quantum fluctuations. Both the QRM phase transition and the PT symmetry breaking are demonstrated in united method. The part of QRM phase transition agrees with the semiclassical solution derived in the previous section. The oscillating frequency of the oscillating phase is also illustrated by a self-consistent equation.

\subsection{Normal Phases and Oscillating in the Regime $g<g_{c}$}

After applying a unitary transformation

$$
U_{n p}=\exp \left[\frac{1}{2} g \eta^{-\frac{1}{2}}\left(a+a^{\dagger}+b+b^{\dagger}\right)\left(\sigma_{+}-\sigma_{-}\right)\right],
$$

to the Hamiltonian in Equation (2), the coherent part of the Hamiltonian can be transformed to

$$
\begin{aligned}
& U_{n p}^{\dagger} H U_{n p} \\
= & \omega_{0}\left(a^{\dagger} a+b^{\dagger} b\right)+\frac{\Omega}{2} \sigma_{x} \\
& +\frac{1}{4} \omega_{0} g^{2}\left(a+a^{\dagger}+b+b^{\dagger}\right)^{2} \sigma_{z}+O\left(\eta^{-\frac{1}{2}}\right) .
\end{aligned}
$$

Because of the extremely large detuning limit $\eta \gg 1$, the last term in the transformed Hamiltonian can be omitted. Additionally, the unitary transformation actually is a infinitesimal one due to $\eta \gg 1$, which would not affect the form of the loss and gain shown as Lindblad operator $\mathcal{L}[\rho]$ in Equation (1). It has been proven in Hwang et al. [30].

In order to demonstrate the property of the normal phase where the qubit eventually stays at the ground state $|\downarrow\rangle$, the qubit degree of freedom can be traced out by averaging the transformed master equation on the ground state of the qubit as

$$
\dot{\rho}_{n p}=-i\left[H_{n p}, \rho_{n p}\right]+\mathcal{L}\left[\rho_{n p}\right]
$$

with

$$
\begin{aligned}
H_{n p} & =\left\langle\downarrow\left|U_{n p}^{\dagger} H U_{n p}\right| \downarrow\right\rangle \\
& =\omega_{0}\left(a^{\dagger} a+b^{\dagger} b\right)-\frac{1}{4} \omega_{0} g^{2}\left(a+a^{\dagger}+b+b^{\dagger}\right)^{2} .
\end{aligned}
$$

and $\rho_{n p}=\left\langle\downarrow\left|U_{n p}^{\dagger} \rho U_{n p}\right| \downarrow\right\rangle$. Obviously, two cross-cavity couples to each other with the coupling constant up to the second order of $g$ and the PT symmetric structure is still preserved. For the mean amplitude $\mathbf{u}=\left(\langle a\rangle,\left\langle a^{\dagger}\right\rangle,\langle b\rangle,\left\langle b^{\dagger}\right\rangle\right)^{T}$, a set of linear equations can be derived from the Equation (15) as $\dot{\mathbf{u}}=\mathbf{L}_{\mathbf{n p}} \mathbf{u}$ with

$$
\mathbf{L}_{\mathbf{n p}}=\left[\begin{array}{cccc}
-i \delta \omega-\kappa & i \gamma_{n} & i \gamma_{n} & i \gamma_{n} \\
-i \gamma_{n} & i \delta \omega-\kappa & -i \gamma_{n} & -i \gamma_{n} \\
i \gamma_{n} & i \gamma_{n} & -i \delta \omega+\kappa & i \gamma_{n} \\
-i \gamma_{n} & -i \gamma_{n} & -i \gamma_{n} & i \delta \omega+\kappa
\end{array}\right]
$$

$\delta \omega=\omega_{0}-\gamma_{n}$ and $\gamma_{n}=\frac{1}{2} \omega_{0} g^{2}$. The eigenvalues of the $\mathbf{L}_{\mathbf{n p}}$ are $l_{n p, \pm}^{ \pm}= \pm i \sqrt{A_{n p} \pm \sqrt{B_{n p}}}$ with

$$
\begin{aligned}
A_{n p} & =\omega_{0}^{2}-2 \gamma_{n} \omega_{0}-\kappa^{2}, \\
B_{n p} & =\omega_{0}\left(\omega_{0} \gamma_{n}^{2}+2 \gamma_{n} \kappa^{2}-\kappa^{2} \omega_{0}\right) .
\end{aligned}
$$

Both the PT symmetry breaking and the dissipative cross-cavity QRM phase transition can be illustrated by the eigenvalues $l_{n p, \pm}^{ \pm}$. If the real part of $l_{n p, \pm}^{ \pm}$is smaller than 0 , the total system eventually decay to the trivial solution $\mathbf{u}=(0,0,0,0)^{T}$ which is the exact normal phase of the QRM. This critical point is determined by $A_{n p} \pm \sqrt{B_{n p}}=0$, which can be rewritten as $2 g^{2}=1+\kappa^{2} / \omega_{0}^{2}$ agreeing with the semiclassical solution in the Equation (5). However, the $B_{n p}$ could varies from a positive value to a negative one when crossing another critical point at $B_{n p}=0$ which corresponds to

$$
g^{2}=2\left(\sqrt{\frac{\kappa^{4}}{\omega_{0}^{4}}+\frac{\kappa^{2}}{\omega_{0}^{2}}}-\frac{\kappa^{2}}{\omega_{0}^{2}}\right) .
$$

As shown in the Figure 2, the regime $g<g_{c}$ can be divided into three regions as: $B_{n p}<0$ for normal phase I; $B_{n p}>0$ and $A_{n p} \pm$ $\sqrt{B_{n p}}<0$ for normal phase II; $B_{n p}>0$ and $A_{n p} \pm \sqrt{B_{n p}}>0$ for oscillating phase. Here the oscillating phase emerges due to the competition between the PT symmetry breaking and the QRM phase transition. It is supposed to emerge when $g>g_{c}$ due to the same mechanism which will be discussed in the next subsection.

\subsection{Superradiant Phase and Oscillating Phase in the Regime $g>g_{c}$}

To take into the consideration of the oscillating phase when $g>$ $g_{c}$, a displacement unitary transformation $D[\alpha]=D_{a}[\alpha] D_{b}[\beta]$ with $D_{a}[\alpha]=\exp \left(\alpha a^{\dagger}-\alpha^{*} a\right)$ and $D_{b}[\beta]=\exp \left(\beta b^{\dagger}-\beta^{*} b\right)$

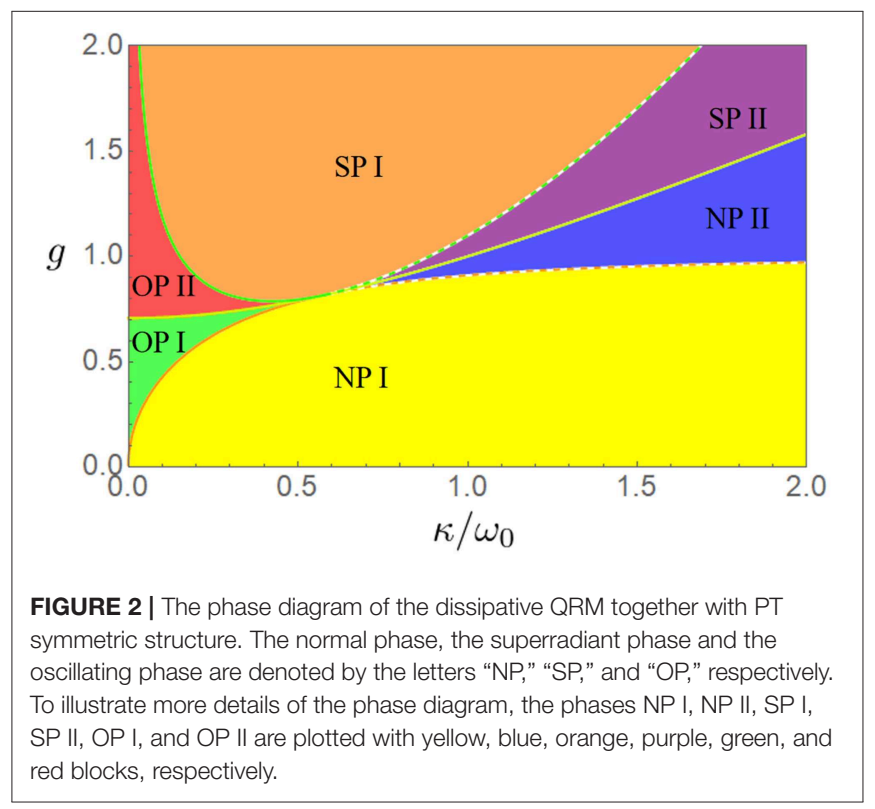


is applied to the Hamiltonian in Equation (2) to displace the cavity modes. The natural choice of the displacements are $\alpha=$ $\pm \frac{g}{1-i \frac{k}{\omega_{0}}} S_{+}$and $\beta= \pm \frac{g}{1+i \frac{k}{\omega_{0}}} S_{-}$in Equation (7) in order to consistent with the semiclassical solution. Here, the fact that the displacement for the first and the second cavity are complex conjugate as $\alpha=\beta^{*}$ reflects the PT symmetric structure.

With the help of the following relations derived from the Equations (4a-4d)

$$
\lambda\left(\alpha_{s}+\alpha_{s}^{*}+\beta_{s}+\beta_{s}^{*}\right)=-\frac{\Omega}{2} \frac{\left\langle\sigma_{x}\right\rangle}{\left\langle\sigma_{z}\right\rangle},\left\langle\sigma_{+}\right\rangle=\left\langle\sigma_{-}\right\rangle=\frac{1}{2}\left\langle\sigma_{x}\right\rangle,
$$

the Hamiltonian can be transformed as

$$
\begin{aligned}
& D^{\dagger}[\alpha] H_{0} D[\alpha] \\
= & \omega_{0}\left(a^{\dagger} a+b^{\dagger} b+2|\alpha|^{2}\right) \\
& \pm \lambda\left\langle\sigma_{x}\right\rangle\left(a+a^{\dagger}\right) \pm \lambda\left\langle\sigma_{x}\right\rangle\left(b+b^{\dagger}\right) \\
& -\lambda\left(a+a^{\dagger}+b+b^{\dagger}\right) \sigma_{x}+\frac{\Omega}{2}\left(\sigma_{z} \pm \frac{\left\langle\sigma_{x}\right\rangle}{\left\langle\sigma_{z}\right\rangle} \sigma_{x}\right) .
\end{aligned}
$$

Clearly, the \pm sign corresponds to the bifurcation of the steady solutions. We only consider the + case in the following discussion. The displacement unitary transformation not only displace the mean field amplitude of the cavity modes out of zero, but also change the magnetic field on the qubit from the $z$-axis to a new direction determined by $\sigma_{z} \pm \frac{\left\langle\sigma_{x}\right\rangle}{\left\langle\sigma_{z}\right\rangle} \sigma_{x}$. When the meanfield amplitude of $\left\langle\sigma_{x}\right\rangle$ is a nonzero value at superradiant phase, the unitary transformation applied previously should along the new direction as

$$
U_{s p}=\exp \left[\frac{1}{2} g \eta^{-\frac{1}{2}} \cos ^{2} \theta\left(a+a^{\dagger}+b+b^{\dagger}\right)\left(\tau_{+}-\tau_{-}\right)\right],
$$

with $\tau_{z}=\cos \theta \sigma_{z}-\sin \theta \sigma_{x}, \tau_{x}=\sin \theta \sigma_{z}+\cos \theta \sigma_{x}$, and $\tan \theta=$ $\frac{\left\langle\sigma_{x}\right\rangle}{\left\langle\sigma_{z}\right\rangle}$. After neglecting the constant term, the effective transformed master equation is obtained as

$$
\dot{\rho}_{s p}=-i\left[H_{s p}, \rho_{s p}\right]+\mathcal{L}\left[\rho_{s p}\right]
$$

with

$$
H_{s p}=\omega_{0}\left(a^{\dagger} a+b^{\dagger} b\right)-\frac{1}{4} \omega_{0} \frac{g_{c}^{4}}{g^{2}}\left(a+a^{\dagger}+b+b^{\dagger}\right)^{2} .
$$

In the same method with the one applied for normal phase for the mean amplitude $\mathbf{u}=\left(\langle a\rangle,\left\langle a^{\dagger}\right\rangle,\langle b\rangle,\left\langle b^{\dagger}\right\rangle\right)^{T}$, a set of linear equations can be derived from the Equation (15) as $\dot{\mathbf{u}}=\mathbf{L}_{\mathbf{s p}} \mathbf{u}$. If we replace the $\gamma_{n}$ with $\gamma_{s}=\frac{1}{2} \omega_{0} \frac{g_{c}^{4}}{g^{2}}$ in Equation (17), the eigenvalues of the $\mathbf{L}_{\mathbf{s p}}$ can be obtained immediately as $l_{s p, \pm}^{ \pm}=$ $\pm i \sqrt{A_{s p} \pm \sqrt{B_{s p}}}$ with

$$
A_{s p}=\omega_{0}^{2}-2 \gamma_{s} \omega_{0}-\kappa^{2}
$$

$$
B_{s p}=\omega_{0}\left(\omega_{0} \gamma_{s}^{2}+2 \gamma_{s} \kappa^{2}-\kappa^{2} \omega_{0}\right)
$$

As we can see in the regime $g>g_{c}$, the effective coupling is inversely proportional to $g^{2}$ instead of being proportional to $g^{2}$ when $g<g_{c}$, which results in quite different phase transitions in comparison with the ones in the regime $g<g_{c}$. As shown in the Figure 2, the regime $g>g_{c}$ can be also divided into three regions as: $B_{s p}<0$ for superradiant phase I; $B_{s p}>0$ and $A_{s p} \pm \sqrt{B_{s p}}<0$ for superradiant phase II; $B_{s p}>0$ and $A_{s p} \pm \sqrt{B_{s p}}>0$ for oscillating phase.

\subsection{Determine the Oscillating Frequency at the Oscillating Phase}

In the last two subsections, the existence of the oscillating phase are verified by the effective master equation. However, the oscillating frequency is not yet determined. Actually the oscillating solution only introduce an additional frequency shift $\omega$ into the Hamiltonian $H_{0}$ as $H_{0}+\omega\left(a^{\dagger} a+b^{\dagger} b+\sigma_{z}\right)$. Such shift would not change the form of effective master equation as Equation (15) in the regimes $g<g_{c}$ and Equation (24) in the regimes $g>g_{c}$. In those oscillating phases, the imaginary parts of the pure imaginary eigenvalues of the $\mathbf{L}_{\mathbf{n p}}$ or $\mathbf{L}_{\mathbf{s p}}$ actually are the oscillating frequency, which indicates that the oscillating frequency is actually determined by a self-consistent equation. In this sense, the self-consistent equation is $\omega= \pm \sqrt{A_{n p}^{\prime} \pm \sqrt{B_{n p}^{\prime}}}$ in the regimes $g<g_{c}$ and $\omega= \pm \sqrt{A_{s p}^{\prime} \pm \sqrt{B_{s p}^{\prime}}}$ in the regimes $g>g_{c}$ with

$$
\begin{aligned}
& A_{n p}{ }^{\prime}=\left(\omega_{0}^{\prime}\right)^{2}-2 \gamma_{n}^{\prime} \omega_{0}^{\prime}-\kappa^{2}, \\
& B_{n p}{ }^{\prime}=\omega_{0}^{\prime}\left(\omega_{0}^{\prime}\left(\gamma_{n}^{\prime}\right)^{2}+2 \gamma_{n}^{\prime} \kappa^{2}-\kappa^{2} \omega_{0}^{\prime}\right) .
\end{aligned}
$$

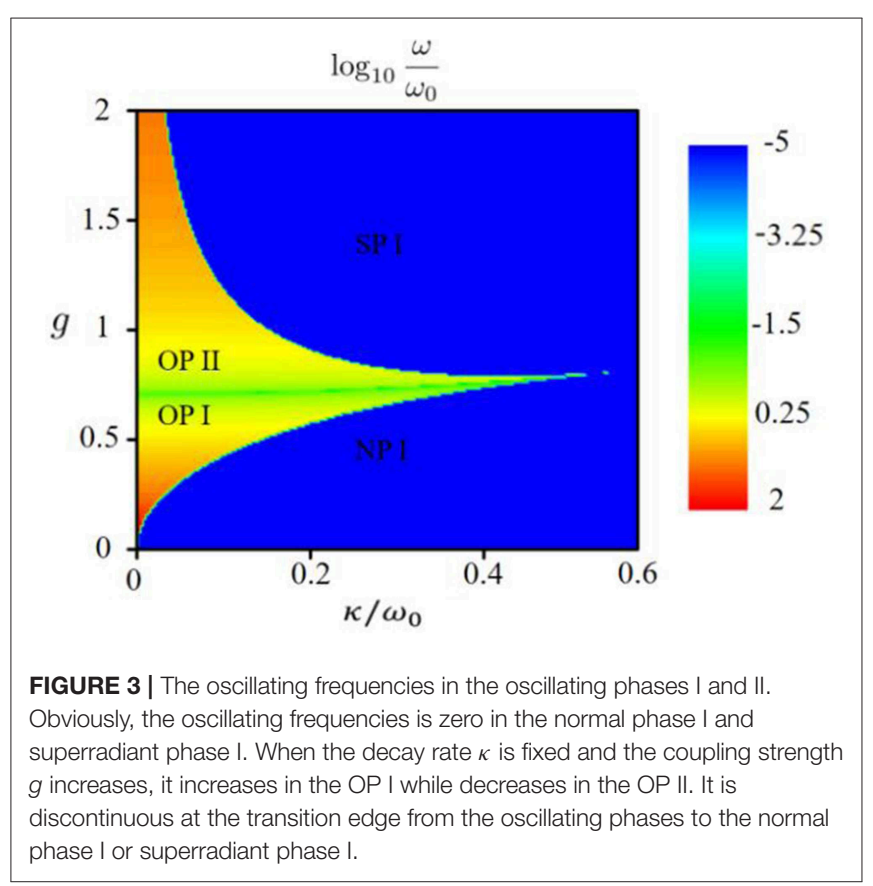


and

$$
\begin{aligned}
& A_{s p}{ }^{\prime}=\left(\omega_{0}^{\prime}\right)^{2}-2 \gamma_{s}^{\prime} \omega_{0}^{\prime}-\kappa^{2}, \\
& B_{s p}{ }^{\prime}=\omega_{0}^{\prime}\left(\omega_{0}^{\prime}\left(\gamma_{s}^{\prime}\right)^{2}+2 \gamma_{s}^{\prime} \kappa^{2}-\kappa^{2} \omega_{0}^{\prime}\right) .
\end{aligned}
$$

The oscillating frequency solved by the above self-consistent equations are shown in Figure 3. As we can see, the behaviors of the oscillating frequencies in the oscillating phase I (OP I) and oscillating phase II (OP II) are quite different. When the decay rate $\kappa$ is fixed and the coupling strength $g$ increases, it increases in the OP I while decreases in the OP II. The oscillating frequencies is almost independent of the decay rate. Another important feature of the oscillating frequencies is discontinuity at the transition edge from the oscillating phases to the normal phase I (NP I) or superradiant phase I (SP I) shown in the phase diagram. It results in the discontinuity of the order parameters in Equations (11-12) which corresponds to the first order quantum phase transition.

\section{CONCLUSION}

In this paper, we investigate the plentiful phase transitions and phase diagram of the cross-cavity quantum Rabi model together with PT symmetric structure. We find that the competition between the quantum Rabi phase transition and the PT symmetry breaking results in plentiful phase transitions and phase diagram. The normal phase and superradiant phase emerging in the previous dissipative quantum Rabi model still exist in our system. The effective coupling is inversely proportional to $g^{2}$ in the superradiant phase instead of being proportional to $g^{2}$ in the normal phase. In contrast, the PT asymmetric phase and symmetric phase can also affect the dissipative quantum Rabi model. For the PT asymmetric phase, the total system has steady state when the time tends to infinite because the energy will eventually dissipate into the surrounding environment. When the PT asymmetric phase

\section{REFERENCES}

1. Rabi II. On the process of space quantization. J Phys Rev. (1936) 49:324-8. doi: 10.1103/PhysRev.49.324

2. Rabi II. Space quantization in a gyrating magnetic field. Phys Rev. (1937) 51:652-4. doi: 10.1103/PhysRev.51.652

3. Todorov Y, Andrews AM, Sagnes I, Colombelli R, Klang P, Strasser G, et al. Strong light-matter coupling in subwavelength metal-dielectric microcavities at terahertz frequencies. Phys Rev Lett. (2019) 102:186402. doi: 10.1103/PhysRevLett.102.186402

4. Ballester D, Romero G, García-Ripoll JJ, Depp F, Solano E. Quantum Simulation of the Ultrastrong-Coupling Dynamics in Circuit Quantum Electrodynamics. Phys Rev X. (2012) 2:1421. doi: 10.1103/PhysRevX.2.021007

5. Pedernales JS, Lizuain I, Felicetti S, Romero G, Lamata L, Solano E. Quantum Rabi model with trapped ions. Sci Rep. (2015) 5:15472. doi: 10.1038/srep15472

6. Muravev VM, Andreev IV, Kukushkin IV, Schmult S, Dietsche W. Observation of hybrid plasmon-photon modes in microwave transmission of coplanar microresonators. Phys Rev B. (2011) 83:1-5. doi: 10.1103/PhysRevB.83.075309 changes to a symmetric one, the gain and loss of the system can be rebalanced and the original dissipative quantum Rabi model behaves like a decay free Rabi model. It means that except the normal phase and superradiant phase existing in the previous dissipative quantum Rabi model, an oscillating phase emerges in the current system, which exactly attributed from the PT symmetric structure. Since the oscillating frequencies is no longer continuous at the transition edge from the oscillating phases to the normal phases or the superradiant phases, a first order phase transition emerges due to the discontinuity of the order parameters inherited from the oscillating frequencies. It may shed light on the research of the first order quantum phase transition.

\section{DATA AVAILABILITY}

All datasets generated for this study are included in the manuscript/supplementary files.

\section{AUTHOR CONTRIBUTIONS}

Z-RG tutored and participated in the writing of some of the papers. LZ tutored the revised paper. JL tutored the calculation and urged the revision of the thesis. NW conducts deductive calculations, plots, and participates in thesis writing.

\section{FUNDING}

This work was supported by National Natural Science Foundation of China (Grants Nos. 11575058, 11434011, 11935006, and 11975095).

\section{ACKNOWLEDGMENTS}

We also thank Dr. Zhihai Wang for helpful discussion.
7. Scalari G, Maissen C, Turcinkova D, Hagenmüller D, De Liberato S, Ciuti C, et al. Ultrastrong coupling of the cyclotron transition of a $2 \mathrm{D}$ electron gas to a THz metamaterial. Science. (2012) 335:1323-6. doi: 10.1126/science.1216022

8. Kéna-Cohen S, Maier SA, Bradley DDC. Ultrastrongly coupled excitonpolaritons in metal-clad organic semiconductor microcavities. Adv Opt Mater. (2013) 1:827-33. doi: 10.1002/adom.201300256

9. Askenazi B, Vasanelli A, Delteil A, Todorov Y, Andreani LC, Beaudoin G, et al. Ultra-strong light-Cmatter coupling for designer Reststrahlen band. N J Phys. (2014) 16:043029. doi: 10.1088/1367-2630/16/4/043029

10. Crespi A, Longhi S, Osellame R. Photonic realization of the quantum Rabi model. Phys Rev Lett. (2012) 108:163601. doi: 10.1103/PhysRevLett.108.163601

11. Rodríguez-Lara BM, Soto-Eguibar F, Cárdenas AZ, Moya-Cessa HM. A classical simulation of nonlinear Jaynes-Cummings and Rabi models in photonic lattices. Opt Express. (2013) 21:12888. doi: 10.1364/OE.21.012888

12. Wolf FA, Kollar M, Braak D. Exact real-time dynamics of the quantum Rabi model. Phys Rev A. (2012) 85:1956-61. doi: 10.1103/PhysRevA.85.053817

13. Zhong H, Xie Q, Batchelor M, Lee C. Analytical eigenstates for the quantum Rabi model. Physics. (2013) 46:295-8. doi: 10.1088/1751-8113/46/41/415302 
14. Gardas B, Dajka J. New symmetry in the Rabi model. J Phys A Math Theor. (2013) 46:493-4. doi: 10.1088/1751-8113/46/26/265302

15. Jaynes ET, Cummings FW. Comparison of quantum and semiclassical radiation theories with application to the beam maser. Proc IEEE. (1963) 51:89-109.

16. Niemczyk T, Deppe F, Huebl H, Menzel EP, Schwarz MJ, Garcia-Ripoll JJ, et al. Circuit quantum electrodynamics in the ultrastrong-coupling regime. Nat Phys. (2010) 6:772-6. doi: 10.1038/nphys1730

17. Todorov Y, Andrews AM, Colombelli R, De Liberato S, Ciuti C, Klang P, et al. Ultrastrong light-matter coupling regime with polariton dots. Phys Rev Lett. (2010) 105:196402. doi: 10.1103/PhysRevLett.105.196402

18. Geiser M, Castellano F, Scalari G, Beck M, Nevou L, Faist J. Ultrastrong coupling regime and plasmon polaritons in parabolic semiconductor quantum wells. Phys Rev Lett. (2012) 108:106402. doi: 10.1103/PhysRevLett.108.106402

19. Cacciola A, Di SO, Stassi R, Saija R, Savasta S. Ultrastrong coupling of plasmons and excitons in a nanoshell. ACS Nano. (2012) 8:11483-92. doi: 10.1021/nn504652w

20. Baust A, Hoffmann E, Haeberlein M, Schwarz MJ, Eder P, Goetz J, et al. Ultrastrong coupling in two-resonator circuit QED. Phys Rev B. (2016) 93:214501. doi: 10.1103/PhysRevB.93.214501

21. Dlaz PF, Garcla-Ripoll JJ, Peropadre B, Orgiazzi JL, Yurtalan MA, Belyansky $\mathrm{R}$, et al. Ultrastrong coupling of a single artificial atom to an electromagnetic continuum in the nonperturbative regime. Nat Phys. (2017) 13:39-43. doi: 10.1038/nphys3905

22. Yoshihara F, Fuse T, Ashhab S, Kakuyanagi K, Saito S, Semba K. Superconducting qubit-oscillator circuit beyond the ultrastrong-coupling regime. Nat Phys. (2017) 13:44-7. doi: 10.1038/nphys3906

23. Gu X, Kockum AF, Miranowicz A, Liu Y-X, Nori F. Microwave photonics with superconducting quantum circuits. Phys Rep. (2017) 718-9:1-102. doi: 10.1016/j.physrep.2017.10.002

24. Hwang MJ, Puebla R, Plenio MB. Quantum phase transition and universal dynamics in the Rabi model. Phys Rev Lett. (2015) 115:180404. doi: 10.1103/PhysRevLett.115.180404

25. Hwang MJ, Plenio MB. Quantum phase transition in the finite jaynes-cummings lattice systems. Phys Rev Lett. (2016) 117:123602. doi: 10.1103/PhysRevLett.117.123602

26. Puebla R, Hwang MJ, Plenio MB. Excited-state quantum phase transition in the Rabi model. Phys Rev A. (2016) 94:023835. doi: 10.1103/PhysRevA.94.023835

27. Bakemeier L, Alvermann A, Fehske H. Quantum phase transition in the Dicke model with critical and noncritical entanglement. Phys Rev A. (2012) 85:043821. doi: 10.1103/PhysRevA.85.043821

28. Ashhab S. Superradiance transition in a system with a single qubit and a single oscillator. Phys Rev A. (2013) 87:679-85. doi: 10.1103/PhysRevA.87. 013826

29. Larson J, Irish EK. Some remarks on 'superradiant' phase transitions in light-matter systems. J Phys A Math Theor. (2017) 50:174002. doi: 10.1088/1751-8121/aa65dc

30. Hwang MJ, Rabl P, Plenio MB. Dissipative phase transition in the open quantum Rabi model. Phys Rev A. (2018) 97:013825. doi: 10.1103/PhysRevA.97.013825

31. Ai Q, Li Y, Zheng H, Sun CP. Quantum anti-Zeno effect without rotating wave approximation. Phys Rev A. (2010) 81:82. doi: 10.1103/PhysRevA.81.042116

32. Bender CM, Boettcher S. Real spectra in non-Hermitian Hamiltonians having PT symmetry. Phys Rev Lett. (1998) 80:5243.

33. Bender CM, Weir DJ. scrPT phase transition in multidimensional quantum systems. J Phys A Math Theor. (2007) 45:425303. doi: $10.1088 / 1751-8113 / 45 / 42 / 425303$
34. Mostafazadeh A. Pseudo-hermiticity versus PT symmetry: the necessary condition for the reality of the spectrum of a non-Hermitian Hamiltonian. J Math Phys. (2002) 43:205-14. doi: 10.1063/1.1418246

35. Peng B, Odemir SK, Lei F, Monifi F, Gianfreda M, Long GL, et al. Paritytime-symmetric whispering-gallery microcavities. Nat Phys. (2014) 10:394-8. doi: $10.1038 /$ nphys 2927

36. Chang L, Jiang X, Hua S, Yang C, Wen J, Jiang L, et al. Parity-time symmetry and variable optical isolation in active-passive-coupled microresonators. Nat Photon. (2014) 8:524-9. doi: 10.1038/nphoton.2014.133

37. $\mathrm{Wu} \mathrm{JH}$, Artoni M, Rocca GCL. Non-Hermitian degeneracies and unidirectional reflectionless atomic lattices. Phys Rev Lett. (2014) 113:123004. doi: 10.1103/PhysRevLett.113.123004

38. Li C, Jin L, Song Z. Non-Hermitian interferometer: unidirectional amplification without distortion. Phys Rev A. (2017) 95:022125. doi: 10.1103/PhysRevA.95.022125

39. Ramezani H, Kottos T, Elganainy R, Christodoulides DN. Unidirectional nonlinear PT-symmetric optical structures. Phys Rev A. (2010) 82:1015-8. doi: 10.1103/PhysRevA.82.043803

40. Lin Z, Ramezani H, Eichelkraut T, Kottos T, Cao H, Christodoulides DN, et al. Unidirectional invisibility induced by PT-symmetric periodic structures. Phys Rev Lett. (2011) 106:213901. doi: 10.1103/PhysRevLett.106.213901

41. Guo A, Salamo GJ, Duchesne D, Morandotti R, Volatier-Ravat M, Aimez V, et al. Observation of PT-symmetry breaking in complex optical potentials. Phys Rev Lett. (2009) 103:093902. doi: 10.1103/PhysRevLett.103.093902

42. Jing H, Odemir SK, Geng Z, Zhang J, Lu XY, Peng B, et al. Optomechanicallyinduced transparency in parity-time-symmetric microresonators. Sci Rep. (2015) 5:9663. doi: 10.1038/srep09663

43. Peng B, Odemir SK, Rotter S, Yilmaz H, Liertzer M, Monifi F, et al. Loss-induced suppression and revival of lasing. Science. (2014) 346:328-32. doi: 10.1126/science. 1258004

44. Feng L, Wong ZJ, Ma RM, Wang Y, Zhang X. Single-mode laser by parity-time symmetry breaking. Science. (2014) 346:972-5. doi: 10.1126/science.1258479

45. Hodaei H, Miri MA, Heinrich M, Christodoulides DN, Khajavikhan M. Parity-time-symmetric microring lasers. Science. (2014) 346:975-8. doi: $10.1126 /$ science. 1258480

46. Jing H, Odemir SK, Lu XY, Zhang J, Yang L, Nori F. PT-symmetric phonon laser. Phys Rev Lett. (2014) 113:053604. doi: 10.1103/PhysRevLett.113.053604

47. He B, Yang L, Xiao M. Dynamical phonon laser in coupled active-passive microresonators. Phys Rev A. (2016) 94:031802. doi: 10.1103/PhysRevA.94.031802

48. West CT, Kottos T, Prosen T. PT-symmetric wave Chaos. Phys Rev Lett. (2010) 104:054102. doi: 10.1103/PhysRevLett.104.054102

49. LV XY, Jing H, Ma JY, Wu Y. PT-symmetry-breaking Chaos in optomechanics. Phys Rev Lett. (2015) 114:253601. doi: 10.1103/PhysRevLett.114.253601

50. Liu ZP, Zhang J, Odemir SK, Peng B, Jing H, Lu XY, et al. Metrology with PT -symmetric cavities: enhanced sensitivity near the PT -phase transition. Phys Rev Lett. (2016) 117:110802. doi: 10.1103/PhysRevLett.117.110802

Conflict of Interest Statement: The authors declare that the research was conducted in the absence of any commercial or financial relationships that could be construed as a potential conflict of interest.

Copyright (c) 2019 Wang, Gong, Lu and Zhou. This is an open-access article distributed under the terms of the Creative Commons Attribution License (CC BY). The use, distribution or reproduction in other forums is permitted, provided the original author(s) and the copyright owner(s) are credited and that the original publication in this journal is cited, in accordance with accepted academic practice. No use, distribution or reproduction is permitted which does not comply with these terms. 